\title{
Aplicação da análise fatorial na caracterização dos acidentes de trânsito na BR-364 em Rondônia
}

\section{Application of factor analysis in the characterization of traffic accidents on BR-364 in Rondônia}

\author{
Emanuel Henrique Azevedo de Castro ${ }^{1}$, Vania Corrêa Mota ${ }^{1 *}$
}

\begin{abstract}
RESUMO
O artigo tem como objetivo analisar e identificar variáveis que mais contribuam com suas cargas fatoriais na caracterização dos acidentes de trânsito ocorridos por ingestão de álcool e/ou substâncias psicoativas na BR-364 no trecho do Estado de Rondônia, bem como, localizar os principais pontos de ocorrência, a fim de, buscar medidas para minimizar os diversos problemas causados pelos acidentes. Os dados utilizados refere-se ao período entre 01/01/2016 até 30/11/2020, e contém os registros de acidentes devido a ingestão de álcool e/ou substâncias psicoativas da rodovia BR-364, um total de 472 registros. A análise multivarida fatorial foi utilizada para grupar e caracterizar as variáveis correlacionadas em fatores. Verificou-se que os maiores pontos de ocorrência de acidentes estão situados nos municípios mais populosos do estado. A Análise Fatorial resultou em três fatores, de um total inicial de 12 variáveis. Conclui-se que a metodologia utilizada foi adequada e com base nos fatores encontrados, órgãos públicos, podem focar seus esforços de melhoria e contribuição para estudos da origem dos acidentes rodoviários, com o benefício da análise detalhada por quilômetro.
\end{abstract}

Palavras-chave: Acidentes rodoviários; Análise multivariada; Análise fatorial exploratória; Ingestão de álcool, Políticas Públicas.

\begin{abstract}
The article has objective analyze and identify variables that most contribute to their factor loads in the characterization of traffic accidents caused by alcohol and/or psychoactive substances on the BR-364 in the stretch of the State of Rondônia, as well as locate the main points of occurrence, in order to seek measures to minimize the various problems caused by accidents. The data used in this work refer to the period from 01/01/2016 to 11/30/2020, and contains records of accidents due to alcohol and/or psychoactive substances ingestion on the BR-364 highway, a total of 472 records. Multivariate factor analysis was used to group and characterize the variables correlated into factors. It has been observed that the highest accident occurrence points are located in the most populous municipalities in the state. Factor Analysis resulted in three factors, out of an initial total of 12 variables. It is concluded that the methodology used was adequate and based on the factors found, public agencies can focus their efforts on improving and contributing to studies of the origin of road accidents, with the benefit of a detailed analysis per kilometer.
\end{abstract}

Keywords: Road Accidents; Multivariate Analysis; Exploratory Factor Analysis; Alcohol Intake; Public Policy.

\footnotetext{
${ }^{1}$ Fundação Universidade Federal de Rondônia (UNIR), Departamento Acadêmico de Matemática e Estatística (DAME), Ji-Paraná, RO, Brasil. *E-mail: vaniamota33@gmail.com
} 


\section{INTRODUÇÃO}

No Brasil, os acidentes de trânsito, são fatores importantes de morbimortalidade geral, sendo considerados atualmente, como verdadeiros problemas de saúde pública (OMS, 2004; ABREU et al. 2010). A Organização Mundial da Saúde (OMS) divulgou estudos que, acidentes de trânsito no mundo, vitimam 1,25 milhões de pessoas por ano, acumulando vítimas fatais principalmente em países pobres (OMS, 2015). Conforme a OMS (2015), os países de baixa ou média renda acumulam 90\% das mortes no trânsito enquanto somam $54 \%$ dos veículos do mundo.

Os acidentes de trânsito no Brasil tendem a se agravar, devido à expansão da frota de veículos automotores que o país vem apresentando desde o final do século passado (PEA, 2015). Estima-se que, o aumento do fluxo de veículos nos grandes centros e rodovias, reflete diretamente na deterioração dessas vias de circulação. Além de pressionar os índices de acidentes nas rodovias do país, em especial as rodovias federais fiscalizadas pela Polícia Rodoviária Federal - PRF, onde boa parte dos óbitos ocorrem (IPEA, 2015; PRF, 2020).

Diante do cenário relacionado a acidentes veiculares, o CNT (2019), apresentou um relatório com o custo financeiro dos acidentes rodoviários entre os anos de 2009 e 2019, o resultado foi de 156 bilhões, o que é aproximadamente o volume de investimentos governamentais em infraestrutura e manutenção das atividades da Polícia Rodoviária Federal - PRF no mesmo período, num total de R\$172,6 bilhões. Dessa forma, muitos pesquisadores têm estudado os fatores que influenciam nos acidentes de trânsito nas rodovias federais brasileiras (BALBO et al., 2010; OLIVEIRA et al., 2018) para auxiliar as políticas públicas na implementação de ações de mitigação dos níveis de gravidade de acidentes.

De modo geral, alguns fatores podem estar associados aos acidentes de trânsito, como por exemplo, dirigir sob efeito de drogas e/ou álcool, no entanto, Almeida (2013) e Alves \& Oliveira (2013) mencionam que, infelizmente, o Brasil ainda está em estágio inicial de reconhecer o comportamento de dirigir alcoolizado como um grave problema de saúde pública.

Particularmente, a problemática dos acidentes de trânsito, são tema de pesquisa de diversos autores, tais como, Minervi (2013) que aplicou a Análise Fatorial no conjunto de variáveis contidas no Boletim de Acidentes de Trânsito (BAT), para obter fatores 
existentes e reduzir o quantitativo de variáveis contidas no BAT, entre outros. Balbo et al. (2010) que ultilizou a análise fatorial exploratória buscando encontrar relações internas em registros de acidentes de trânsito com o objetivo de agrupar variáveis relacionadas que pudessem caracterizar melhor as causas de ocorrência dos acidentes na rodovia.

Diante do exposto, entende-se que, analisar fenômenos que envolvam uma grande quantidade de informações variáveis relacionadas as causas dos acidentes, exige a aplicação das técnicas da Estatística Multivariada. Dentre as técnicas destaca-se a Análise Fatorial que busca agrupar e caracterizar as variáveis correlacionadas em fatores, resumindo assim a grande quantidade de variáveis envolvidas nos acidentes, além de identificar os pontos da rodovia com as principais causas e tipos de acidentes (MINGOTI, 2013).

Neste contexto, esse artigo tem como objetivo analisar e identificar variáveis que mais contribuam com suas cargas fatoriais na caracterização dos acidentes de trânsito ocorridos por ingestão de álcool e/ou substâncias psicoativas na BR-364 no trecho do Estado de Rondônia, bem como, localizar os principais pontos de ocorrência, a fim de, fornecer informações úteis para o órgão responsável, para que possam buscar ações para minimizar os diversos problemas causados pelos acidentes no trecho da BR-364 em estudo, e assim, desenvolver e aplicar medidas de correção em trechos específicos da rodovia.

\section{MATERIAL E MÉTODOS}

\section{Dados utilizados}

Os dados utilizados neste trabalho, estão disponíveis no site da Polícia Rodoviária Federal (PRF, 2020). Tomou-se como base para este trabalho os anos compreendidos no período entre 01/01/2016 até 30/11/2020. Os dados contém os registros de acidentes de todas as rodovias federais do Brasil, portanto, foi necessário filtrar os dados de acordo com a rodovia (BR-364), depois por estado (Rondônia) e por ultimo pela causa do acidente (Ingestão de álcool e/ou substâncias psicoativas) para que se chegasse na delimitação do tema proposto no trabalho resultando um total de 472 registros de acidentes.

Dentre as várias variáveis contidas neste banco de dados as seguintes foram selecionadas para a análise: "Ano", "Data inversa", "Dia da semana", "Horário", "KM”, 
"Município", "Tipo do acidente”, "Classificação do acidente”, "Condições meteorológicas", "Tipo da pista", "Traçado da via" e "Uso do solo". A variável "Data inversa" foi transformada na variável "Mês", por meio de agrupamento de informações, além dela a variável "Horário", também passou por agrupamento, cujo os registros de acidente de 00:00h até 05:59h foram agrupados em "Madrugada", de 06:00h até 11:59h agrupados em "Manhã", de 12:00h até 17:59h agrupados em "Tarde" e de 18:00h até 23:59h agrupados em "Noite".

Feito isso, resultou-se em um banco de dados com 12 variáveis, destas, apenas duas são quantitativas, "Ano" e "KM", as demais variáveis são qualitativas. Logo, para a aplicação da análise multivariada de dados, foi necessário categorizar as variáveis qualitativas dando uma ordem numérica as observações de cada uma delas. As variáveis qualitativas, que não possuiam uma ordem lógica (Ex.: Janeiro, Fevereiro, Março, Abril...), foram organizadas de acordo com o percentual de ocorrência, categorizando suas observações desde o menor percentual de ocorrência até a maior. A variável Município foi enumerada desde a primeira cidade do estado de Rondônia transpassada pela rodovia até a última, e a váriável "Uso do Solo" passou a ser chamada de Perímetro (Urbano e Rural).

\section{Análise Fatorial Exploratória}

Segundo Mingoti (2013), seja $X_{p x 1}$ um vetor aleatório, com $n$-variáveis observáveis, com um vetor de média $\mu$, onde, $\mu=\left(\mu_{1}, \mu_{2}, \ldots, \mu_{n}\right)$, matriz de covariância $\Sigma_{p x p}$ e matriz de correlação $P_{p x p}$. Sejam $Z_{i}=\left[\left(X_{i}-\mu_{i}\right) / \sigma_{i}\right]$ variáveis originais padronizadas, onde $\mu_{i}$ e $\sigma_{i}$ representem respectivamente a média e o desvio padrão da variável $X_{i}, i=1,2 \ldots, p$. Neste caso, a matriz de correlação $P$ é a matriz de covariâncias do vetor aleatório $Z=\left(Z_{1}, Z_{2}, \ldots, Z_{p}\right)^{\prime}$.

O modelo fatorial construído a partir da matriz de correlação teórica $P_{p x p}$ é formalizado conforme as equações dadas em 1 e 2 abaixo:

$$
\begin{gathered}
Z_{1}=L_{11} F_{1}+L_{12} F_{2}+\ldots+L_{1 m} F_{m}+\varepsilon_{1} \\
Z_{2}=L_{21} F_{1}+L_{22} F_{2}+\cdots+L_{2 m} F_{m}+\varepsilon_{2} \\
\vdots \quad \vdots \quad \quad \vdots \quad \vdots \quad \vdots \quad \vdots \quad \vdots \\
Z_{p}=L_{p 1} F_{1}+L_{p 2} F_{2}+{ }^{\cdots}+L_{p m} F_{m}+\varepsilon_{p}
\end{gathered}
$$

Sendo $m \leq p$.

O modelo linear (1), em notação matricial é expresso por: 


$$
D(X-\mu)=L F+\varepsilon
$$

onde,

$$
\begin{gathered}
(X-\mu)_{p x 1}=\left[\begin{array}{cc}
X_{1}- & \mu_{1} \\
X_{2}- & \mu_{2} \\
\vdots & \vdots \\
X_{p}- & \mu_{p}
\end{array}\right], \varepsilon_{p x 1}=\left[\begin{array}{c}
\varepsilon_{1} \\
\varepsilon_{2} \\
\vdots \\
\varepsilon_{p}
\end{array}\right], F_{m x 1}=\left[\begin{array}{c}
F_{1} \\
F_{2} \\
\vdots \\
F_{m}
\end{array}\right], L_{p x m}=\left[\begin{array}{cccc}
l_{11} & l_{12} & \ldots & l_{1 m} \\
l_{21} & l_{22} & \ldots & l_{2 m} \\
\vdots & \vdots & \ddots & \vdots \\
l_{p 1} & l_{p 2} & \ldots & l_{p m}
\end{array}\right], \\
D_{p x p}=\left[\begin{array}{ccccc}
1 / \sigma_{1} & 0 & 0 & \cdots & 0 \\
0 & 1 / \sigma_{2} & 0 & \cdots & 0 \\
\vdots & \vdots & \ddots & \cdots & \vdots \\
\vdots & \vdots & \vdots & \ddots & \vdots \\
0 & 0 & 0 & \cdots & 1 / \sigma_{p}
\end{array}\right] .
\end{gathered}
$$

Assim, $F_{m x 1}$, é um vetor aleatório com $m$ fatores, também chamados de variáveis latentes, que descrevem os elementos da população estudada e não são observáveis, $1 \leq$ $m \leq p$, ou seja não podem ser medidos a priori (MINGOTI, 2013). Nesse sentido o modelo de análise fatorial assume que as variáveis $Z$ estão correlacionadas linearmente com novas variáveis aleatórias $F_{j}, j=1,2 \ldots, m$, que deverão ser analisadas e identificadas pelo pesquisador. O Vetor $\varepsilon_{p x 1}$ é um vetor de erros aleatórios correspondentes aos erros de medidas e variação de $Z_{i}$, que não é explicada pelos fatores comuns $F_{j}, j=1,2 \ldots, m$, incluídos no modelo.

O coeficiente $l_{i j}$, é o coeficiente da $i$-ésima variável padronizada $Z_{i}$, comumente chamado de peso ou Carga Fatorial (CF), no $J$ - ésimo fator $F_{j}$, e representa o grau de relacionamento linear entre $Z_{i}$ e $F_{j}, j=1,2 \ldots, m$. Os coeficientes $l_{i j}$ ou cargas fatoriais, ficam armazenados na matriz $L_{p x m}$, que é uma matriz de parâmetros (constantes) a serem estimados. Assim de acordo com o modelo linear (1), as informações das $n$-variáveis originais padronizadas $\left(Z_{1}, Z_{2}, \ldots, Z_{n}\right)$ estarão sendo representadas por $(p+m)$ variáveis aleatórias não observáveis, ou seja, $\left(\varepsilon_{1}, \varepsilon_{2}, \ldots, \varepsilon_{p}\right)$ e $\left(F_{1}, F_{2}, \ldots, F_{m}\right)$.

São necessárias determinadas suposições para que se possa operacionalizar a estimação do modelo em (1), seguiu as suposições descritas em Mingoti, (2013).

Para verificar se a matriz de correlação populacional é próxima ou não da matriz identidade recorremos ao Teste de Esfericidade de Bartlett, o qual testa a hipótese de que a matriz de correlação das variáveis $l_{p x p}$ é uma matriz identidade $P_{p x p}$. Caso não se rejeite $H_{0}: P=l$, significa que $\rho_{i j}=0(i \neq j)$, então as variáveis não estão correlacionadas. 
A estatística do teste é dada por (BARTLETT, 1937):

$$
T=-n\left[-\frac{1}{6}(2 p+11)\right]\left[\sum_{j=1}^{p} \ln \left(\hat{\lambda}_{i}\right)\right]
$$

onde $n$ é o tamanho da amostra, $\ln ($.$) denota a função logaritmo neperiano e, \hat{\lambda}_{i}=$ $1,2 \ldots, p)$ são os autovalores da matriz de correlação amostral $R_{p x p}$. Sob a hipótese nula e $n$ grande a estatística do teste tem uma distribuição aproximadamente qui-quadrado $X^{2}$ com $\frac{1}{2} p(p-1)$ graus de liberdade. Para que o modelo de análise fatorial possa ser ajustado, o teste de Bartlett deve rejeitar a hipótese nula (BARTLETT, 1937).

Para medir a adequação da amostra utilizou-se do teste Kaiser-Meyer-Olkin (KMO) e da Medida de Adequação da Amostra (MSA). O índice KMO é dado por (HAIR et al., 2009):

$$
K M O=\frac{\sum_{j \neq i} \sum r_{i j}^{2}}{\sum_{j \neq i} \sum r_{i j}^{2}+\sum_{j \neq i} \sum a_{i j}^{2}}
$$

variando entre 0 e 1 , onde $r_{i j}$ é o coeficiente de correlação entre variáveis e $a_{i j} \mathrm{o}$ coeficiente de correlação parcial, em que, quanto menor as correlações parciais mais o índice se aproxima de 1 . A regra para interpretação dos índices de KMO é que, KMO $\geq$ 0,75 é considerado Bom, 0,5 $\leq \mathrm{KMO}<0,75$ aceitável e $\mathrm{KMO}<0,5$ inaceitável para aplicar a análise fatorial na amostra de dados (KAISER, 1958; PASQUALI, 1998; OLIVEIRA et al., 2018; CORRÊA et al., 2019). Este índice confronta a amplitude das correlações observadas entre as variáveis com a amplitude das correlações parciais, uma vez que esta última aponta o grau de relação entre duas variáveis ignorando a influência das outras.

A Medida de Adequação da Amostra (MSA) é utilizada para quantificar o grau de intercorrelação entre as variáveis e a adequação da análise fatorial, e calculada pela por (HAIR et al., 2009):

$$
M S A=\frac{\sum_{j \neq i} r_{i j}^{2}}{\sum_{j \neq i} r_{i j}^{2}+\sum_{j \neq i} a_{i j}^{2}}
$$

Se o resultado de MSA apresentar um valor baixo, podemos detectar as variáveis responsáveis por esse efeito. A análise do MSA é comparável ao KMO, as variáveis encontradas dentro do domínio inaceitável devem ser excluídas para posteriormente 
aplicar a análise fatorial, ou seja, MAS $\geq 0,75$ é considerado Bom, 0,5 $\leq$ MAS $<0,75$ aceitável e MSA $<0,5$ inaceitável para proceder a análise fatorial sem a remoção desta variável.

Utilizou-se dos critérios descritos em Mingoti (2013) para estimação do número de fatores $m$. A escolha do número de fatores é essencial e tem como objetivo a substituição do conjunto de variáveis originais por fatores, sendo natural que o número de fatores seja inferior ao número de variáveis analisadas (CORRAR et al., 2007). Com isso ao invés de se trabalhar com $100 \%$ da variabilidade total dos dados, analisa-se somente, uma parcela total dos dados explicadas por fatores.

Para verificar a rotação desses fatores aplicou-se o método Varimax, que segundo Fávero et al. (2009) é o método mais utilizado e busca minimizar o número de variáveis que têm altas cargas em um fator, simplificando a interpretação dos fatores.

O modelo fatorial proposto em (2), é semelhante a um modelo de regressão linear múltipla se considerarmos $Z$ como um vetor de respostas, $F$ como vetor de parâmetro do modelo, $\varepsilon$ o vetor de erros aleatórios com média zero e matriz de covariâncias $\Psi$ e $L_{p x m}$ como a matriz com os níveis das $m$ variáveis explicativas, ou seja, $Z=L F+\varepsilon$. Tratando o modelo fatorial dessa forma, esse estudo usou o método de regressão, e pode-se estimar o vetor $F$ através do método dos mínimos quadrados ponderados (JOHNSON \& WICHERN, 2002).

Para uma melhor visualização do perfil dos acidentes por ingestão de álcool e/ou substâncias psicoativas na BR-364 no trecho do Estado de Rondônia, os dados foram agrupados em um único arquivo. Por meio da utilização de planilha eletrônica os dados foram separados e organizados. A aplicação das análises estatísticas multivariada e descritiva, foram desenvolvidas no software R (R CORE TEAM, 2020).

\section{RESULTADOS E DISCUSSÃO}

Preliminarmente aplicou-se a análise de estatítica descritiva, e por meio de alguns gráficos pôde-se conhecer melhor o banco de dados. Com o histograma de distribuição de frequência, disposto na Figura 1, pode-se observar as características contidas na variável "KM", sendo possível identificar os pontos de maior concentração dos acidentes por Ingestão de álcool. Os registros da variável KM incia-se no quilômetro 10 até o quilômetro 972. 
Observando a Figura 1, existem vários picos, cujo o primeiro pico da esquerda para a direita corresponde a cidade de Vilhena, seguido por mais dois picos entre os quilômetros 150 e 280 aproximadamente, que correspondem respectivamente aos municípios de Pimenta Bueno e Cacoal. Entre os quilômetros 320 e 360, é identificado o segundo maior pico do histograma correspondente ao município de Ji-Paraná e por últimoentre os quilômetros 700 e 780 aproximadamente o pico mais alto do histograma que corresponde a capital do estado de Rondônia, a cidade de Porto Velho.

Figura 1- Distribuição dos acidentes na BR - 364 entre os municípios de Vilhena e Porto Velho.

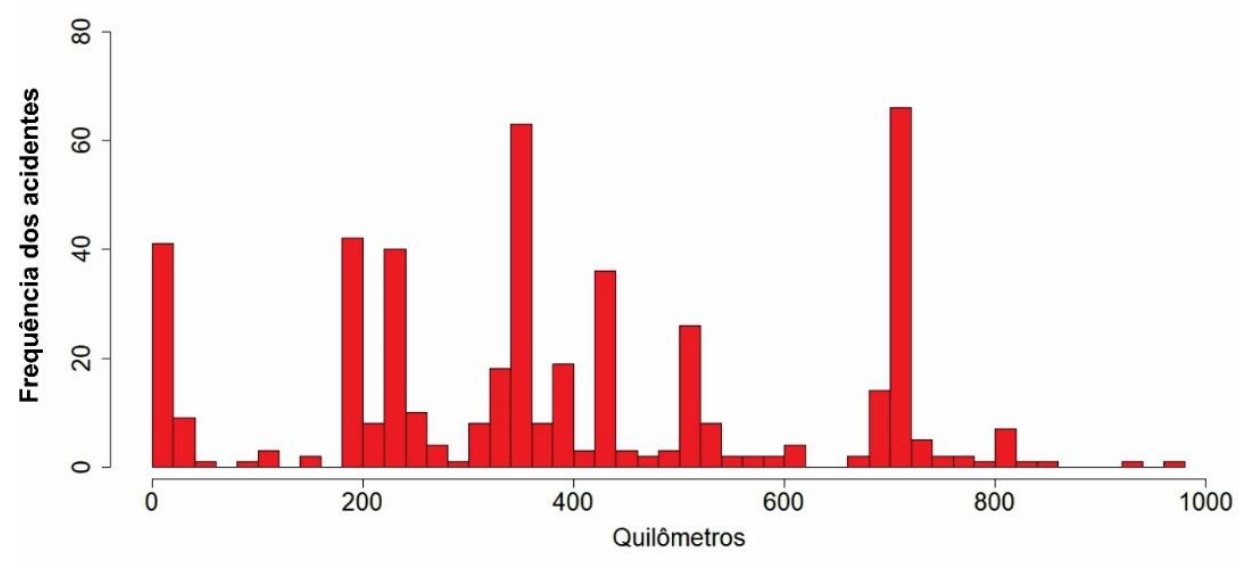

Fonte: Autores (2021)

Verifica-se com base nos dados analisados que os maiores pontos de ocorrência de acidentes estão situados nos municípios mais populosos do estado, o que já pode ser uma justificativa, sendo um fato, o grande índice de veículos em trânsitos nestes municípios.

Conforme Ribeiro et al. (2020) são bem raras as pesquisas brasileiras que analisam a falta de atenção na ocorrência de acidentes de trânsito. No entanto, estudos realizados em outros países/regiões indicam que a negligência do motorista é a principal causa dos acidentes de trânsito. Por exemplo, a National Road Traffic Safety Administration estima que pelo menos $25 \%$ dos acidentes envolvem negligência do motorista (STUTTS, 2001). Essa interferência torna difícil para o motorista reconhecer as informações necessárias para uma direção segura, pois certos eventos, atividades, objetos ou pessoas dentro ou fora do veículo podem induzi-lo a desviar sua atenção. 
Na pesquisa de Ribeiro et al. (2020), a "Falta de atenção" apresentou inclusive um impacto maior, estando associada com 48,66\% dos incidentes. Ou seja, esse fator justifica a grande incidência de acidentes na cidade de Porto Velho, possivelmente por ter muitos veículos e as pessoas dirigirem de certa forma desatenta.

Outro fator importante relacionado com a ocorrência de acidentes automobilísticos de acordo com Ribeiro et al. (2020), é o excesso de velocidade. Conforme a Organização Mundial da Saúde, o veículo motorizado acima do limite da via é o principal responsável para a ocorrência de acidentes com vítimas fatais, representando 50\% das mortes no trânsito em países em desenvolvimento (WHO, 2009).

Para cada aumento de $1 \mathrm{~km} / \mathrm{h}$ acelerado, a incidência de acidentes com vítimas aumentou 3\% reduzindo o risco de morte de 4 a 5\% (ANDRADE \& MELLO-JORGE, 2013). O uso de cinto de segurança com menos frequência nos bancos dianteiro e traseiro, a desobediência de regras de trânsito, a adoção de velocidades superiores ao limite permitido nas vias e a associação entre a direção de veículos automotores e o consumo de álcool contribuem para o maior número de óbitos (MALTA et al., 2016).

Na Figura 2, estão representados via gráfico de barras, o percentual de acidentes de trânsito causado por Ingestão de álcool em cada município entre os anos de 2016 a 2020.

Figura 2- Percentual de acidentes em cada munícipio do trecho da BR 364 estudado.

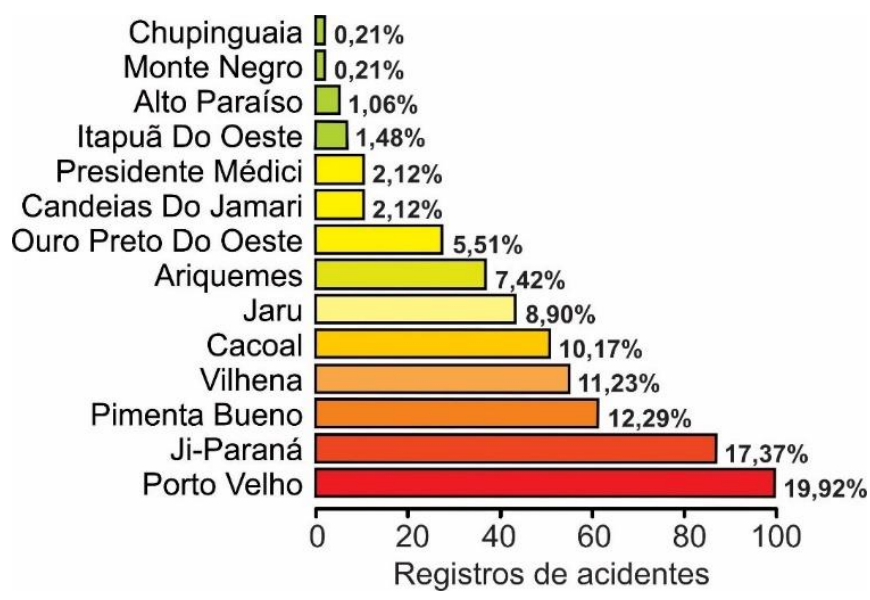

Fonte: Autores (2021)

Analisando a Figura 2, pode-se confirmar o que já foi mencionado anteriormente, a capital Porto Velho foi o município com maior indice de acidentes, com um percentual de 19,92\%, quando comparado com demais cidades que tiveram registros de ocorrência. 
Seguido pelos municípios de Ji-Paraná 17,37\%, Pimenta Bueno 12,39\%, Vilhena 11,23\%, Cacoal $10,17 \%$ e Jaru $8,9 \%$. Os municípios com menor indice de acidentes foram Chupinguaia e Monte Negro com $00,21 \%$ respectivamente.

Para Lima (2007) no altíssimo ranking de mortalidade e morbidade por acidentes de trânsito, o Brasil se destaca devido aos altos índices de casos envolvendo o consumo de bebidas alcoólicas. Portanto, a urgência em discutir e implementar medidas restritivas pode reduzir significativamente esses índices. Nesse sentido, França, Espanha, Japão e outros países têm adotado medidas de controle do uso de álcool, inclusive contra a direção veemente sob o efeito do álcool, e têm obtido sucesso significativo na redução da mortalidade por acidentes de trânsito.

Infelizmente, não se pode ignorar que hoje, no Brasil, o uso de álcool é fortemente incentivado por meio de propagandas de larga escala voltadas para os jovens, pois a própria indústria percebeu o foco das mensagens publicitárias. Em muitos casos, o forte aumento do consumo de bebidas alcoólicas (principalmente cerveja) representa um sério desafio para a implementação de ações que visem reduzir a incidência e mortalidade por acidentes de trânsito e violência urbana (LIMA, 2007).

WHO (2007), rebate que, em comparação com pessoas que bebem enquanto dirigem, os motoristas bêbados têm uma tendência clara de aumentar o risco de acidentes. Com base neste problema, é necessário promulgar a Lei $\mathrm{n}^{\circ} 11.705$ chamada Lei da Proibição de 2008, e atualizá-la em 2022, para determinar a tolerância zero ao teor de álcool no sangue de motoristas de veículos automotores, e ainda criminalizar e punir os motoristas cujo sangue contenha 6 decigramas ou mais de álcool por litro no sangue, ou 0,34 ml ou mais de álcool em seu corpo, conforme medido por um teste de etilômetria (LARANJEIRA \& ROMANO, 2004; LIMA, 2007; MOURA et al., 2009; BRASIL, 2012).

Em relação aos horários do dia com maior ocorrência de acidentes, temos disposto na Figura 3 cada período do dia e seus respectivos percentuais de ocorrência. Como já foi mencionado anteriormente a variável "Horário", passou por um agrupamento de observações, cujo os registros de acidente de 00:00h até 05:59h foram agrupados em "Madrugada", de 06:00h até 11:59h agrupados em "Manhã", de 12:00h até 17:59h agrupados em "Tarde" e de 18:00h até 23:59h agrupados em "Noite".

Entre os períodos citados, o período da noite expressa a maior frequência de acidentes de trânsito causado por Ingestão de álcool e/ou substâncias psicoativas com 
45,3\%, na contra mão o período da manhã obteve o menor indíce com $12,5 \%$ dos registros.

Figuras 3- Percentual de registros de acidente por período do dia.

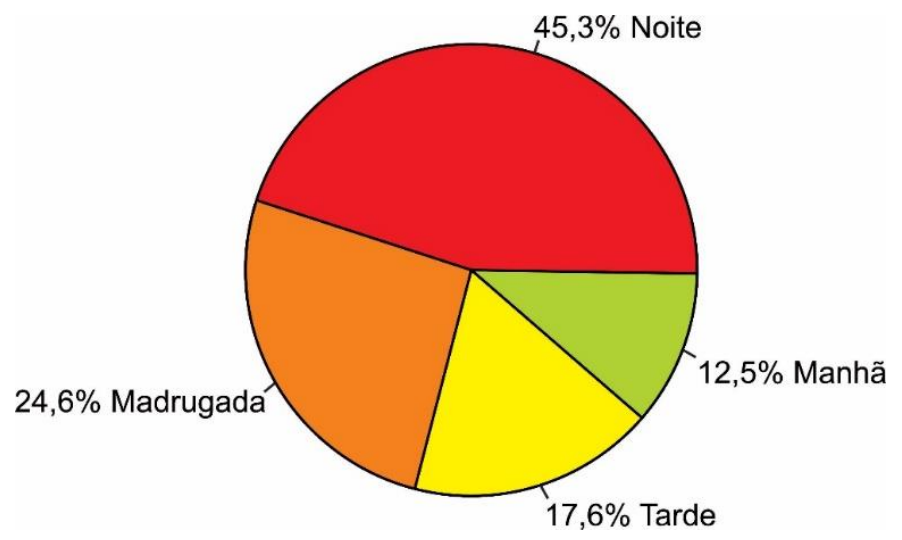

Fonte: Autores (2021)

É de extrema necessidade e urgência a implementação de políticas públicas de educação no trânsito para prevenção de acidentes de trânsito. Além disso, os órgãos fiscalizadores precisam adotar medidas rígidas, principalmente no controle de velocidade, treinamento dos motoristas e verificação das condições dos veículos, isso não induz apenas a redução do número de acidentes, mas também auxilia a reduzir a gravidade para diminuir o impacto. As ações como melhorar as estradas e dar atenção especial aos ciclistas e aos motociclistas, que, infelizmente nos últimos anos, o número de suas frotas e a taxa de participação em acidentes, com aumento do número de mortos e feridos têm aumentado, também precisam ser adotadas (RIBEIRO et al., 2014; INSTITUTO DE PESQUISA ECONÔMICA APLICADA; POLÍCIA RODOVIÁRIA FEDERAL, 2015; SILVA et al., 2015; VILAS BOAS \& SILVA, 2015).

Andrade \& Mello-Jorge (2001) realizaram um estudo na cidade de Londrina no Paraná, contabilizaram que nos diferentes anos de estudo, a distribuição das vítimas por horário de acidentes é muito semelhante, sendo sua maior concentração à noite (18h às 23h59) e à tarde (12h às 17h59). Observaram no ano de 1996, que os acidentes ocorridos à tarde e à noite tiveram em média maior número de vítimas, sendo que nos dias úteis, a maioria das vítimas acidentou-se no horário das $18 \mathrm{~h}$ às $19 \mathrm{~h}$. Indicando que essa situação pode ser causada pelo cansaço, que é maior no final do dia, e também pelo alto tráfego naquele horário (ANDRADE \& MELLO-JORGE, 2001). 
Bastos et al. (2005), relata que normalmente, a noite é o horário com maior incidência de vítimas, pois neste período, devido a múltiplos fatores relacionados ao meio ambiente (baixa visibilidade) e aos usuários das vias públicas (excesso de velocidade), os semáforos não são respeitados e os acidentes costumam ser mais graves. $\mathrm{O}$ elevado número de vítimas à noite e aos finais de semana deve servir de indicador para o desenvolvimento e implementação de planos intersetoriais de prevenção de acidentes e de divulgação de campanhas de educação para o trânsito. É de extrema importância a adoção e implantação dessas campanhas educativas, de preferência, que esclareçam os reais riscos do uso de álcool e direção, mostrando os fatores ambientais que dificultam muitas vezes a direção de veículos automotores ao entardecer (penumbra), a noite e aos demais comportamentos de risco para acidentes ocorrerem.

A Figura 4 representa o percentual dos acidentes que geraram vítimas ou não. Pode-se constatar que 65,3\% dos acidentes que foram causados por Ingestão de álcool e/ou substâncias psicoativas geraram vítimas feridas, 29,9\% dos acidentes não foram registrados vítimas e 4,4\% dos acidentes foram registrados vítimas que vieram a óbito, e em apenas $0,4 \%$ das ocorências registradas a presença ou ausência de vítimas foi ignorada no boletim.

Figuras 4- Percentual de vítima entre os acidentes registrados.

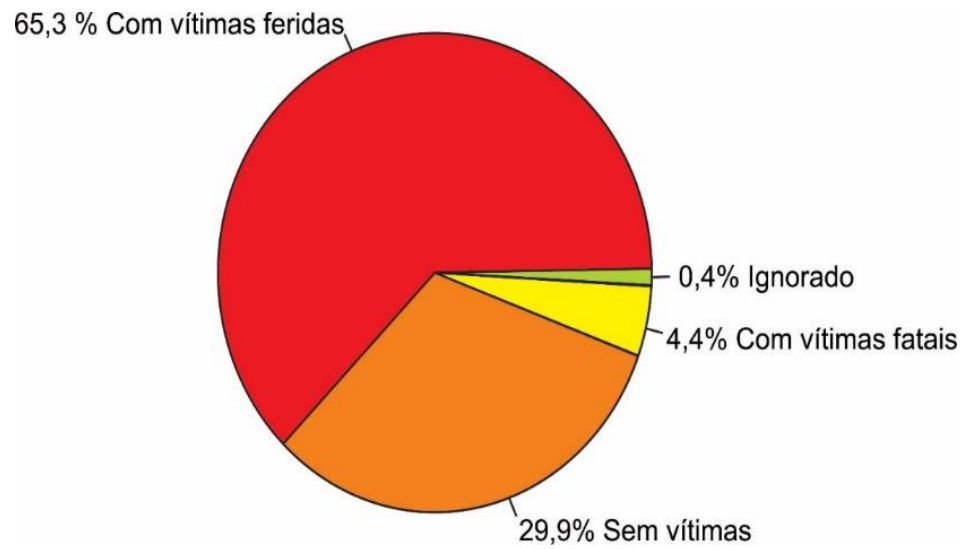

Fonte: Autores (2021)

Ao analisar a variável "Tipo de acidente", identificou os principais tipos de acidente que mais obtiveram registros conforme a Figura 5. A colisão traseira foi a mais comum entre os demais tipos de acidentes registrado com $21,19 \%$ do total de acidentes, seguido por saída de leito carroçável com $13,77 \%$, que consiste na saída do veículo da pista de circulação, colisão lateral 10,38\%, colisão com objeto estático 9,32\% e colisão 
transversal com 8,26\% do total. Contrariamente os tipos de acidentes com menores números de registros foram os com "Atropelamento de animal", "Colisão com bicicleta", "Colisão com objeto em movimento" e "Danos eventuais", todos com um percentual de $0,21 \%$ de ocorrência ente os registros.

Figuras 5- Tipos de acidentes registrados na BR 364 do KM 10 ao 972.

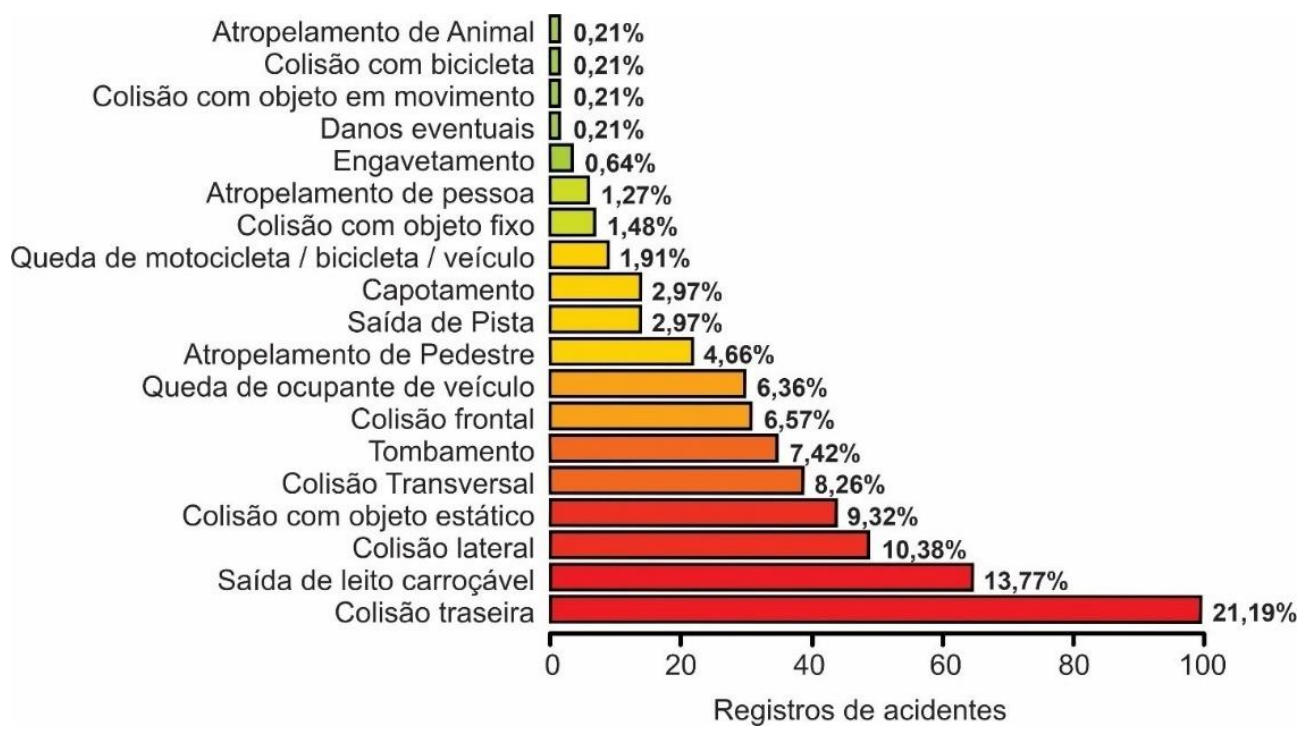

Fonte: Autores (2021)

Mattox et al. (2000), relata que em países desenvolvidos, boa parte dos ferimentos fatais e não fatais causados por acidentes de trânsito, ocorrem na sua maioria em jovens, sendo que, $61 \%$ das pessoas acidentadas com idade abaixo de 45 anos são acometidas com mortes por traumatismo e mais de $30 \%$ são hospitalizadas.

Melhorias como a sinalização, sistema de tráfego e de vias públicas, iluminação, são fundamentais para a redução de acidentes de trânsito (MARSON \& THOMSON, 2001; MURRAY, 1998). Outro fator importante é a fiscalização do trânsito, que deve ser intensificada, facilitando a punição dos motoristas alcoolizados e infratores, fazendo com que haja uma diminuição no número de acidentes e vítimas de acidentes na BR 364 no trecho de Vilhena a Porto Velho em Rondônia.

Após o tratamento dos dados pela estatística descritiva, iniciou-se a análise fatorial aplicando-se o testes de normalidade univariada e multivariada. Para o teste de normalidade univariado, foi aplicado o teste de Shapiro-Wilk e o teste de KolmogorovSmirnov (DeGROOT \& SCHERVISH, 2002; LOPES et al., 2021). Diante dos resultado, 
a estimação do modelo fatorial ortogonal foi análisada pelo método de componentes principais (ARAUJO et al, 2008;CORRÊA, et al., 2019).

A análise fatorial foi aplicada duas vezes na planilha contendo o banco de dados, porém, apenas a segunda análise fatorial foi interpretada devido aos baixos indices gerados pelos testes de KMO (Kaiser-Meyer-Olkin) de MAS (Medida de Adequação da Amostra). Seguindo o critério de corte em que MSA < 0,5 é inaceitável para proceder a análise fatorial, foi necessário remover as seguintes variáveis, MÊS, HORA, CLIMA, PISTA e PERÍMETRO. Após o corte das variáveis, pode-se verificar novamente as estatísticas do Teste de esfericidade de Bartlett, e observou-se que a nova matriz de correlação, não é uma matriz identidade ao nível de significância de 5\%, dessa forma, pode-se afirmar que o conjunto de dados é aceitável para o uso da técnica.

Utilizando o Teste Scree representado pela Figura 6, pode-se identificar o melhor número de fatores a serem extraídos do banco de dados antes de analisarmos a comunalidade das variáveis. Verificou-se que os três primeiros pontos se qualificam como os fatores a serem considerados na análise, considerando ainda o fato de que, o critério da raiz latente conservar todos os fatores com autovalor maior que 1 (um) na análise.

Figura 6- Teste Scree para análise de componentes.

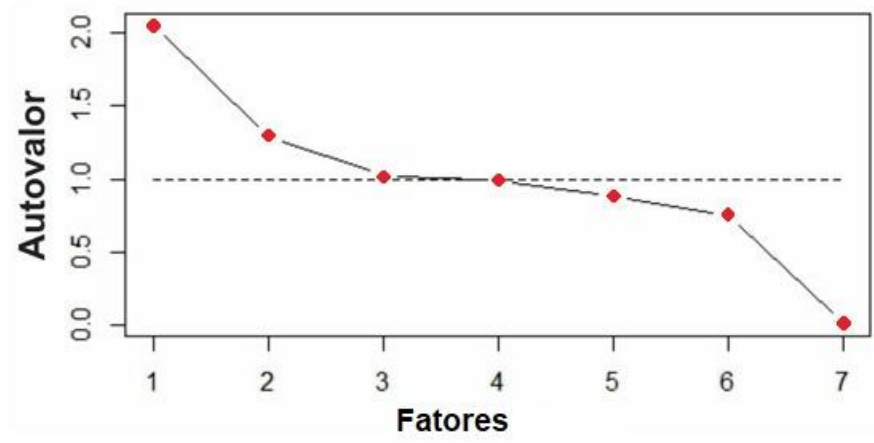

Fonte: Autores (2021)

Estes três fatores representam $63 \%$ da variância total de banco de dados. Os 3 fatores da análise fatorial foram obtidos pelo método das componentes principais e rotacionados pelo critério de rotação VARIMAX. As cargas fatoriais juntamente com a comunalidade de cada variável são apresentadas na Tabela 1. 
Tabela 1. Rotação ortogonal da matriz fatorial.

\begin{tabular}{|c|c|c|c|c|c|c|c|}
\hline \multirow[b]{2}{*}{ Variáveis } & \multicolumn{3}{|c|}{$\begin{array}{l}\text { Antes da Rotação } \\
\text { Cargas Fatoriais }\end{array}$} & \multirow[t]{2}{*}{ h2 } & \multicolumn{3}{|c|}{$\begin{array}{c}\text { Depois da Rotação VARIMAX } \\
\text { Cargas Fatoriais }\end{array}$} \\
\hline & Fator 1 & Fator 2 & Fator 3 & & Fator 1 & Fator 2 & Fator 3 \\
\hline ANO & 0,01 & 0,75 & 0,17 & 0,60 & 0,1 & 0,76 & 0,07 \\
\hline DIA & $-0,26$ & 0,37 & $-0,43$ & 0,40 & $-0,22$ & 0,34 & $-0,48$ \\
\hline KM & 0,99 & 0,08 & 0,00 & 0,98 & 0,99 & $-0,03$ & 0,01 \\
\hline MUNICÍPIO & 0,98 & 0,08 & 0,00 & 0,98 & 0,99 & $-0,03$ & 0 \\
\hline TIPO & 0,05 & $-0,72$ & $-0,03$ & 0,53 & $-0,03$ & $-0,72$ & 0,06 \\
\hline CLASSIFICAÇÃO & 0,14 & $-0,22$ & $-0,01$ & 0,07 & 0,11 & $-0,24$ & 0,02 \\
\hline \multirow[t]{6}{*}{ TRAÇADO } & $-0,13$ & 0,01 & 0,90 & 0,82 & $-0,13$ & 0,14 & 0,89 \\
\hline & & & & Itovalor & 2,040 & 1,290 & 1,029 \\
\hline & \multicolumn{4}{|c|}{ Proporção de variância } & 0,290 & 0,190 & 0,150 \\
\hline & \multicolumn{4}{|c|}{ Variância acumulada } & 0,290 & 0,480 & 0,630 \\
\hline & \multicolumn{4}{|c|}{ Proporção Explicada } & 0,468 & 0,296 & 0,236 \\
\hline & \multicolumn{4}{|c|}{ Proporção Acumulada } & 0,468 & 0,764 & 1 \\
\hline
\end{tabular}

*h2 (comunalidade).

Fonte: Autores (2021)

O fator de número 1 que contém as variáveis "KM" e "MUNICÍPIO" tende a ser um fator geral com cargas significativamente altas nas variáveis, sendo assim, este explica a maior quantia da variância. Os fatores de número 2 que contém as variáveis "ANO" e “TIPO” junto ao fator de número 3 com apenas a variável "TRAÇADO” explicam porções respectivamente menores de variância e são baseados na quantia residual de variância, o objetivo de rotacionar a matriz fatorial é redistribuir a variância dos primeiros fatores com os demais, a fim de tornar a interpretação mais simples e significativa.

Especificamente, nos resultados da análise fatorial rotacionada, o fator 1 explica $29 \%$ da variância total, seguido pelos fatores 2 e 3 que explicam $19 \%$ e $15 \%$ respectivamente. Na Figura 7 temos a representação gráfica dos valores da variância comum (comunalidade) e da variância única (ou específica) de cada variável.

Verificou-se na Figura 7 que as variáveis "KM" e "MUNICÍPIO" são as que mais compartilham suas variâncias, sendo que $98 \%$ de cada uma delas é compartilhado com as outras variáveis que permaneceram na análise. Ao contrário da varável "CLASSIFICAÇÃO" que compartilha apenas 7\% de sua variância, neste caso seria possível considerar a remoção desta variável, porém ela será mantida na pesquisa, para uma melhor interpretação dos fatores. 
Figura 7- Detalhe da comunalidade e da variância específica.

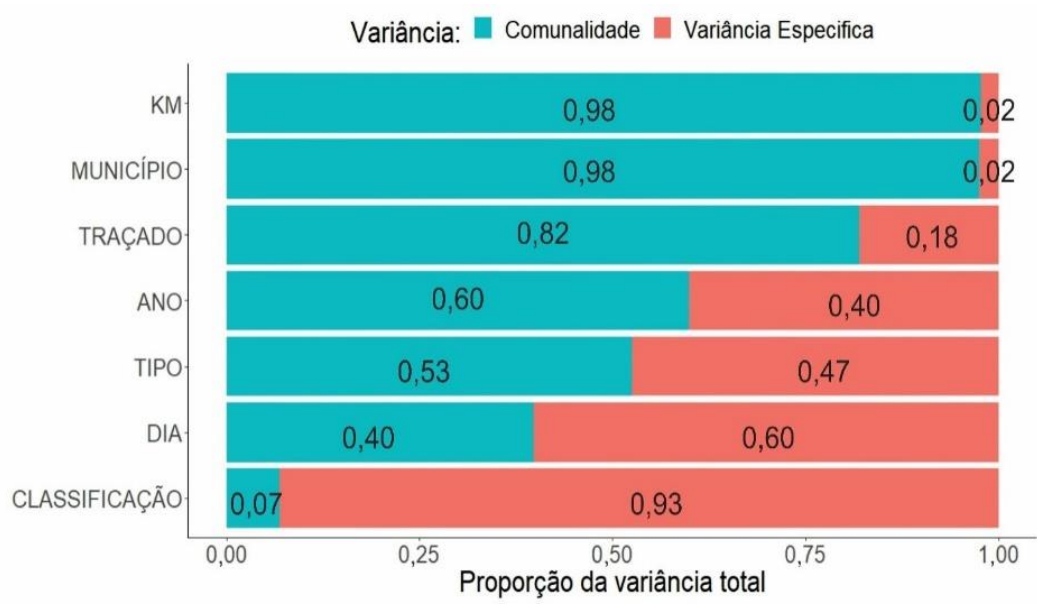

Fonte: Autores (2021)

Portanto a partir da interpretabilidade dos fatores apresentados na Tabela 7, foi possível caracterizar três fatores distintos como, o fator 1 como "Trecho de ocorrência", o fator 2 como "Característico cronológico" e o fator 3 como "Traçado da via" pelo fato deste último conter apenas essa variável. Por meio da aplicação de análise fatorial exploratória e com o uso do critério VARIMAX para a rotação dos fatores, pode-se caracterizar os fatores que melhor explicassem os registros de acidentes no trecho do Estado de Rondônia da rodovia BR-364.

\section{CONCLUSÃO}

Concluiu-se que, a Análise Fatorial se mostrou eficiente na redução da variabilidade do banco de dados. A capital Porto Velho foi o município que apresentou o maior índice de acidentes devido a ingestão de álcool e/ou substâncias psicoativas, com um percentual de 19,92\%. Seguido pelos municípios de Ji-Paraná 17,37\%, Pimenta Bueno 12,39\%, Vilhena 11,23\%, Cacoal 10,17\% e Jaru 8,9\%. Os municípios com menor indice de acidentes foram Chupinguaia e Monte Negro com 0,21\% respectivamente.

Pode-se constatar também que $65,3 \%$ dos acidentes que foram causados por Ingestão de álcool e/ou substâncias psicoativas geraram vítimas feridas, 29,9\% dos acidentes não foi registrado vítimas e 4,4\% dos acidentes foi registrado vítimas que vieram a óbito e em apenas $0,4 \%$ das ocorências registradas a presença ou ausência de vítimas foi ignorada no boletim. A Análise Fatorial resultou em três fatores, de um total 
inicial de 12 variáveis após a rotação dos fatores, "Trecho de ocorrência", "Característico cronológico" e "Traçado da via", representando 63\% da variabilidade total do banco de dados.

Com os resultados obtidos e com base nos fatores encontrados, órgãos públicos, podem focar seus esforços de melhoria e contribuição para estudos da origem dos acidentes rodoviários, com o benefício da análise detalhada por quilômetro. Políticas públicas podem ser traçadas gerando informações para os cidadãos dos trechos rodoviários que apresentam maior perigo de acidente. Entende-se assim, que os objetivos da pesquisa foram alcançados, demonstrando que a metodologia utilizada foi adequada, levando a obter resultados relevantes para a organização de pesquisas futuras no intuito de estudar medidas para que diminuam os acidentes no tráfego de veículos da rodovia BR-364 no trecho do Estado de Rondônia.

\section{AGRADECIMENTOS}

Os autores agradecem ao Grupo de Pesquisa em Modelos Estatísticos, Matemáticos e Fuzzy - MEMF.

\section{REFERENCIAS}

ABREU, A. M. M. et al. Uso de álcool em vítimas de acidentes de trânsito: estudo do nível de alcoolemia. Rev. Latino-Am. Enfermagem, Ribeirão Preto, v.18, n. spe, p. 513-520, 2010.

ALMEIDA, N. D. A saúde no Brasil, impasses e desafios enfrentados pelo Sistema Único de Saúde - SUS.Revista Psicologia e Saúde, Campo Grande, v. 5, n. 1, p. 1-9, 2013.

ALVES, V. S; OLIVEIRA, I. M. S. Atenção à saúde de usuários de álcool e outras drogas no Brasil: convergência entre a saúde pública e os direitos humanos. Revista de Direito Sanitário, São Paulo, v. 13, n. 3, p. 9-32, 2013.

ANDRADE, S. M.; MELLO-JORGE, M. H. P. Acidentes de transporte terrestre em Município da Região Sul do Brasil. Revista Saúde Pública, 2001; 35: 318-20.

ARAUJO, V. M.; MOTA, V. C.; LIMA, I. B. de. Uma abordagem aplicada de componentes principais no estudo do desenvolvimento da mesorregião metropolitana de Belo Horizonte, MG. In: XLVI CONGRESSO DA SOCIEDADE BRASILEIRA DE ECONOMIA, ADMINISTRAÇÃO E SOCIOLOGIA RURAL, Anais[...] Acre, 2008. p. 1-15.

BALBO, F. A. N. et al. Estudo dos Acidentes na BR-277 Por Meio da Análise Fatorial. p. 1022-1023, 2010. 
BARTLETT, M. S. Properties of sufficiency and statistical tests. Proceedingsofthe Royal Society of London, serie A, London, v.160, p. 268-282, 1937.

BASTOS, Y. G. L.; ANDRADE, S. M.; SOARES, D. A. Características dos acidentes de trânsito e das vítimas atendidas em serviço pré-hospitalar em cidade do Sul do Brasil, 1997/2000. Centro de Ciências da Saúde, Universidade Estadual de Londrina, Londrina, Brasil, 2005.

BRASIL. Lei $n^{\circ} 12.760$, de 20 de dezembro de 2012. Altera a Lei no 9.503, de 23 de setembro de 1997, que institui o Código de Trânsito Brasileiro. Diario Oficial da União 2012; 21 dez.

CORRAR, L. J.; PAULO, E.; FILHO, J. M. D. ANÁLISE MULTIVARIADA para os cursos de Administração, Ciências Contábeis e Economia. 1. ed. São Paulo, Atlas/FIPECAFI, 2007.

CORRÊA, A. L.; PRATES, G. A.; GALLI, L. C. do L.; SAVI, A. F. Uma análise de fatores na implementação dos princípios de gestão da qualidade da série de normas iso 9000 em uma organização agroindustrial, Braz. J. of Develop., Curitiba, v. 5, n. 7, p. 10390-10404 jul. 2019.

CNT (2019). Acidentes rodoviários: policiamento, infraestrutura e custo econômico, Brasília, Brasil.

DeGROOT, M. H.; SCHERVISH, M. J. Probability and Statistics. 3rd Edition. Addison Wesley, 2002.

FÁVERO, L. P. L. et al. Análise de Dados - Modelagem Multivariada para Tomada de Decisões. Rio de Janeiro, Campus: Elsevier, 2009.

HAIR JR, J. F.; ANDERSON, R.E.; TATHAN, R.L.; BLACK, W.C.; BABIN, J.B. Análise Multivariada de Dados. Tradução Adonai Schlup Sant' Anna. $6^{\mathrm{a}}$ ed. Porto Alegre: Bookman, 2009.

INSTITUTO DE PESQUISA ECONÔMICA APLICADA; POLÍCIA RODOVIÁRIA FEDERAL. Acidentes de trânsito nas rodovias federais brasileiras: caracterização, tendências e custos para a sociedade. Brasília, DF, 2015. Disponível em:

http://www.ipea.gov.br/portal/index.php?option=com_content\&view=article $\& i d=26277$ . Acesso em: 10 maio 2021.

IPEA. Acidentes de trânsito nas rodovias federais brasileiras: Caracterização, tendências e custos para a sociedade. Instituto de Pesquisa Econômica Aplicada (IPEA), p. 42, 2015.

JOHNSON, R. A.; WICHERN, D. W. Applied multivariate statistical analysis. $3^{\mathrm{a}}$ ed. New York,Prentice Hall, 2002.

KAISER, H. F. The varimax criterion for varimax rotation in factor analysis.

Psychometrika, v. 23, n. 3, p. 187-204, 1958. 
LARANJEIRA, R.; ROMANO, M. Consenso brasileiro sobre políticas públicas do álcool. Ver. Bras. Psiquiatr, v. 26 (Supl.1), p. 68-77, 2004.

LIMA, J. M. B. Palestra Temática Alcoolemia e os Acidentes de Trânsito. In: VII CONGRESSO LATINO AMERICANO DE ACIDENTES DE TRÂNSITO, ABRAMET. Porto Seguro, Bahia; 2007.

LOPES, K. L. M.; MOTA, V. C.; SILVA, A. N. da; SILVA, R. J. de S. da. Estatística experimental aplicada em ensaio de competição de variedades de feijoeiro comum (Phaseolus Vulgaris L.). Braz. J. of Development, v.7, n. 3, p. 30442-30459, 2021.

MALTA, D. C.; ANDRADE, S. S; GOMES, N.; SILVA, M. M.; MORAIS, O. L; REIS, A. A. Injuries from traffic accidents and use of protection equipment in the Brazilian population, according to a population-based study. Ciên. Saúde Coletiva, v. 21, n. 2, p. 399-410. 2016.

MINERVI, N. A. Análise Fatorial e de Séries Temporais Aplicadas às Séries dos Acidentes de Trânsito nas Rodovias Federais Brasileiras. p. 158, 2013.

MINGOTI, S. A. Análise De Dados Através De Métodos De Estatística Multivariada: Uma Abordagem Aplicada. Belo Horizonte: Editora da UFMG; 2013.

MOURA, E. C; MALTA, D. C; MORAIS NETO, O. L; PENNA, G. O.; TEMPORÃO, J. G. Direção de veículos motorizados após consumo abusivo de bebidas alcoólicas, Brasil, 2006 a 2009. Rev. Saúde Pública, v. 43, n. 5, p. 891-894, 2009.

MATTOX, K. L.; FELICIANO, D. V.; MOORE, E. E. Trauma. New York: McGrawHill; 2000.

MARSON, A. C.; THOMSON, J. C. The influenceofpre hospital trauma careon motor vehicle crash mortality. J. Trauma, v. 50, p. 917-21. 2001.

MURRAY, A. The home and school background of young drivers involved in traffic accidents. Accid. Anal Prev., v. 29, p. 169-82. 1998.

OLIVEIRA, D. DE A.; ROSA, G. F.; KEMPA, M. M.; NETO, N. L.; HONGYU, K. Análise fatorial e espacial em dados de crimes e acidentes de trânsito do Estado de Mato Grosso, Biodiversidade, v. 17, n.3, p. 79- 96, 2018.

ORGANIZACIÓN MUNDIAL DE LA SALUDE - OMS. Informe mundial sobre prevención de los traumatismos. causados por el trânsito. Ginebra (SW): OMS; 2004.

OMS. Organização Mundial da Saúde, 2015. Disponível em:

https://nacoesunidas.org/oms-brasil-e-o-pais-com-maior-numero-de-mortes-de-transitopor-habitante-da-america-do-sul/. Acesso em: 6 abr. 2021.

PASQUALI, L. Análise fatorial: um manual teórico-prático. Brasília: Editora da UnB, no prelo, 1998. 
PRF. Polícia Rodoviária Federal. Disponível em: https://www.prf.gov.br/portal/dadosabertos/acidentes/acidentes. Acesso em: 20 set. 2020.

WORLD HEALTH ORGANIZATION (WHO). Beber e Dirigir: Manual de Segurança de Trânsito para Profissionais de Trânsito e Saúde. Genebra: Global Road Safety Partnership; 2007.

WORLD HEALTH ORGANIZATION (WHO). Informe sobre la situación mundial de la seguridad vial: es hora de pasar a la acción. Geneva; 2009. Disponível em: http://www.who.int/mediacentre/ factsheets/ fs310/en/index.html. Acesso em: 8 abr. 2021.

R. A language and environment for statistical computing, $\mathbf{R}$ Development Core Team, R Foundation for Statistical Computing, Vienna, Austria, 2020. Disponível em: http://www.r-project.org. Acesso em: 22 jan. 2021.

RIBEIRO, L. A; PIMENTEL, J. L; RIBEIRO, H; BENEDITO, M. L. H; RIBEIRO, K. L. P. Análise das causas dos acidentes automobilísticos nas rodovias federais da Bahia entre 2014 e 2017/Analysis of the causes of auto accidents on federal highways in Bahia between 2014 and 2017. Ver. Med., (São Paulo), v. 99, n. 1, p. 27-34, 2020.

RIBEIRO, V. P.; DANIEL, J. A.; BLASIUS, L. Acidentes de trânsito envolvendo motociclistas em Cascavel. Revista Brasileira de Segurança Pública, São Paulo, v. 8, n. 1, p. 184-196, 2014.

SILVA, G. J. C. da; MENEZES, L. B.; NEDER, H. D. Qualidade da Malha Rodoviária, Custos Econômicos Associados e Determinantes dos Acidentes de Trânsito no Brasil: Avaliação e proposição de política. Revista de Políticas Públicas, São Luís, v. 19, n. 1, p. 327-347, 2015.

STUTTS, J. C.; REINFURT, D. W; RODGMAN, E.A. The role of driver distraction in crashes: an analysis of 1995-1999 Crashworthiness Data System Data. Annu Proc.

Assoc. Adv. Automat. Med. v. 45, p. 287 - 301, 2001.

VILAS BÔAS, E. F.; SILVA, M. A. da. Trânsito e Segurança Pública: Impactos e Consequências. Humanidades, Montes Claros, v. 4, n. 2, 2015

Recebido em: 15/01/2022

Aprovado em: 16/02/2022

Publicado em: 18/02/2022 